University of Nebraska - Lincoln

DigitalCommons@University of Nebraska - Lincoln

December 1991

\title{
Photoemission and electronic structure of tungsten-based metallic glasses and alloys
}

\author{
M.A. Engelhardt \\ University of Nebraska - Lincoln \\ Sitaram Jaswal \\ University of Nebraska, sjaswal1@unl.edu \\ David J. Sellmyer \\ University of Nebraska-Lincoln, dsellmyer@unl.edu
}

Follow this and additional works at: https://digitalcommons.unl.edu/physicssellmyer

Part of the Physics Commons

Engelhardt, M.A.; Jaswal, Sitaram; and Sellmyer, David J., "Photoemission and electronic structure of tungsten-based metallic glasses and alloys " (1991). David Sellmyer Publications. 114.

https://digitalcommons.unl.edu/physicssellmyer/114

This Article is brought to you for free and open access by the Research Papers in Physics and Astronomy at DigitalCommons@University of Nebraska - Lincoln. It has been accepted for inclusion in David Sellmyer Publications by an authorized administrator of DigitalCommons@University of Nebraska - Lincoln. 


\title{
PHYSICAL REVIEW B \\ CONDENSED MATTER
}

\section{Photoemission and electronic structure of tungsten-based metallic glasses and alloys}

\author{
M. A. Engelhardt, ${ }^{*}$ S. S. Jaswal, and D. J. Sellmyer \\ Behlen Laboratory of Physics and Center for Materials Research and Analysis, \\ University of Nebraska, Lincoln, Nebraska 68588-0111
}

(Received 14 June 1991)

\begin{abstract}
The structure and electronic density of states of intertransition-metal glasses of the form $T_{x} \mathrm{~W}_{1-x}$ have been investigated, where $T=\mathrm{Cu}, \mathrm{Ni}$, and $\mathrm{Co}$. The film samples were prepared by cosputtering in a chamber with a rapidly rotating, water-cooled substrate. Experiments included x-ray diffraction and ultraviolet photoemission at photon energies of 21.2 and $40.8 \mathrm{eV}$, and electronic-structure calculations were performed with a self-consistent linear combination of muffin-tin-orbitals method. For the calculations the structures were simulated by either disordered crystalline or amorphous clusters of 60-64 atoms in a periodically extended supercell. The range of amorphous alloy formation was determined approximately for each alloy series, and it was found that this range was larger when the atomic-radii difference of the two elements is larger. In both the photoemission and theoretical results for the crystalline and amorphous $\mathrm{Cu}-\mathrm{W}$ alloys the $\mathrm{Cu} 3 d$ band is a distinct feature whose position is well reproduced by the calculations. In the $\mathrm{Ni}-\mathrm{W}$ and $\mathrm{Co}-\mathrm{W}$ alloys the $3 d$ and $5 d$ bands overlap significantly. In the case of crystalline Ni-W alloys, the theory gives a good representation of the observed occupied density of states.
\end{abstract}

\section{INTRODUCTION}

Research on intertransition-metal binary alloys has followed from the desire to understand the mechanisms responsible for their physical and electronic properties. Examples of significant problems include the conditions under which amorphous or crystalline phases form, and the fundamental origins of negative temperature coefficients of electrical resistance. At a basic level all of these properties must be controlled by the electronic structure of the valence electrons. ${ }^{1}$ Theoretical understanding of this structure has been difficult to achieve because of the lack of translation symmetry, both for disordered crystalline alloys and amorphous or glassy alloys.

By now a broad variety of binary glasses has been prepared by rapid quenching from the melt or sputtering methods. The subclass of refractory transition-metal alloys is of particular interest because of possible applications in microelectronics or as thin-film coatings. Such materials might be expected to be particularly stable at high temperatures and also in chemically reactive environments. For example, amorphous CuTa ( $a$-CuTa) alloys have been investigated because of their potential use as diffusion barriers. ${ }^{2,3}$ Recently we have studied the electronic structure and electrical resistance of $a-\mathrm{CuTa}$ and crystalline $(c-) c$-CuTa with photoelectron spectroscopy (PES) measurements and performed self-consistent electronic-structure calculations on these alloys. ${ }^{4} \mathrm{~A}$ density-of-states (DOS) calculation using the linear muffin-tin-orbitals (LMTO) method was in good agreement with the PES spectra.

Tungsten films have recently been used for microelectronic circuits. Several studies of the properties of the films ${ }^{5,6}$ and their uses ${ }^{7}$ have been conducted. Elemental tungsten may be deposited by chemical vapor deposition (CVD) by the $\mathrm{WF}_{6}-\mathrm{H}_{2}$ reaction, electron-beam evaporation, and sputtering. Within an integrated circuit, tungsten may be used to lower the resistance of interconnects and for interconnect filling via holes between different circuit levels. It may also be used to modify the semiconductor structures as a diffusion barrier. Using CVD, the tungsten may be selectively deposited on the interconnects without additional masking steps. Tungsten, which has been proven to be less reactive with aluminum and silicon than other metals, forms a harder structure which is less susceptible to damage, more corrosion resistant, and more suitable for higher currents. ${ }^{7,8}$

Due to tungsten's properties, alloys of tungsten are also of interest. Understanding the properties of tungsten alloys becomes crucially important in determining their usefulness compared to elemental tungsten. Therefore in this work we have prepared by sputtering a wide variety of alloys of the form $T_{x} \mathrm{~W}_{1-x}$, where $T=\mathrm{Cu}, \mathrm{Ni}$, and $\mathrm{Co}$. Presented here are compositional data as obtained by $\mathrm{x}$ ray fluorescence, structural data as obtained from $x$-ray diffraction, and electronic-structure information as de- 
rived from ultraviolet PES measurements. These latter data are compared with self-consistent LMTO calculations for selected compositions of amorphous and crystalline structures. We have given earlier brief reports on the atomic and electronic structures of nearby compositions of amorphous and crystalline $\mathrm{CuW}$ alloys. ${ }^{9}$

\section{EXPERIMENTAL METHODS}

\section{A. Sample preparation and characterization}

The $T_{x} \mathrm{~W}_{1-x}$ alloys were prepared by a cosputtering technique as described in our previous paper on CuTa alloys. ${ }^{4}$ Here only a few points will be mentioned that are specific to this study.

Tungsten was sputtered using a radio-frequency (rf) magnetron gun, and the transition metal with a direct current (dc) magnetron gun. The metals were deposited onto substrates and by moving the substrate from one gun to another quickly enough, less than one monolayer of metal was deposited before the substrate moved again. By arranging the substrates onto a rotating carousel (see Ref. 4) the requirements of cosputtering were easily met.

The rotating table was water cooled. Depending upon the substrate, different conditions were observed for the thermal conduction of heat away from the substrate. Substrates of Mylar, glass, and copper sheets were used. For copper, the substrates were held tightly against the carousel and were most likely at the temperature of the cooling water. For glass substrates, the fixtures that hold the substrates to the carousel were bent to "spring load" the glass against the surface of the carousel. It is estimated that the temperatures of the rotating Mylar substrates could be assumed to be somewhere between $100^{\circ} \mathrm{C}$ and $200^{\circ} \mathrm{C}$, the glass substrates probably at temperatures less than $100^{\circ} \mathrm{C}$, and the copper substrates at temperatures less than $50^{\circ} \mathrm{C}$.

The vacuum chamber was pumped to a base pressure of $3 \times 10^{-7}$ Torr before sputtering. During sputtering, the diffusion pump was throttled and prepurified argon gas was admitted to the chamber to a pressure of 7.5 mTorr by an electrically operated valve controlled by a MKS Baratron.

The sputtering rates were determined for the different sources in separate experiments where only a single gun was operated producing elemental films. The thicknesses of the films were calculated after weighing the films. Plots of sputtering rate versus $\mathrm{rf}$ power or dc current were made as calibrations.

The alloys were made across the composition range with six or more different atomic stoichiometries. By varying the sputtering rates, different stoichiometries could be produced. The films were deposited at the highest possible rates within the limitations of the sputtering sources. Samples made in the middle of the composition ranges were deposited at the highest combined rates. It typically took $15-60 \mathrm{~min}$ to produce a $2500 \AA$ thickness. X-ray fluorescence (XRF) measurements were performed to determine the sample stoichiometries and were performed on all 23 of the alloys prepared. The XRF analysis showed the samples were usually richer in tungsten then anticipated on the basis of the individual sputtering gun calibration rates. This occurred in 20 to 23 samples despite repeated checking of the $\mathrm{W}$ calibration. This may have resulted from different sticking coefficients at the substrate for elemental and alloy films. Compositions listed on the $\mathrm{x}$-ray diffraction figures and the PES spectra in the following sections are the XRF-determined values.

The alloys were deposited on glass, Mylar, and copper substrates to facilitate different analysis techniques. The glass substrates provided a smooth background for x-ray diffraction and the Mylar a low background signal for $\mathrm{x}$ ray fluorescence measurements. The three substrates also gave a better chance that if the properties of a particular alloy caused cracking, ${ }^{9}$ for instance, the other substrates would have useable films. PES measurements were made on films deposited on glass or, if the film was flaking or cracking, on copper.

$\mathrm{X}$-ray diffraction was used to determine phase production across the composition range and to see whether amorphous or noncrystalline phases existed. The x-ray diffraction data were obtained with a Rigaku D Max IIB diffractometer fitted with a copper target $(\lambda=1.54 \AA)$. Crystalline peaks were indexed using the $X-R a y$ Powder Data File and Index ${ }^{10}$ and the Crystal Data Determinative Tables. ${ }^{11}$ Pure tungsten films prepared by sputtering were deposited as bcc tungsten with lattice parameter $2.52 \AA$. At times the $\beta-\mathrm{W}$ phase was produced which increased the lattice parameter to $2.56 \AA .{ }^{12}$ Some of the tungsten-rich alloy phases also showed evidence of the $\beta$ $\mathrm{W}$ phase, but no photoemission data on samples with this structure are reported in this paper.

\section{B. Photoelectron spectroscopy measurements}

The major components of the homemade PES apparatus include the ultrahigh-vacuum (UHV) chamber which houses the samples on a rotatable sample holder, an ion gun for cleaning the samples, an electron energy analyzer with an electron multiplier to detect the electrons, and an ultraviolet source located external to the chamber. Light from the discharge source passes through a glass tube into the UHV chamber and strikes the sample. Helmholtz coils mounted outside the chamber provide a field-free region inside the chamber. A few details of the components are given below.

The UHV chamber was pumped by a Perkin Elmer TNB-X DI ion pump. The base pressure is better than $3 \times 10^{-10}$ Torr after a bakeout at $100^{\circ} \mathrm{C}$. The uv source is differentially pumped gas discharge lamp, capable of producing radiation from $\mathrm{He}_{\mathrm{I}}(584 \AA, 21.22 \mathrm{eV})$ and He II (304 $\AA, 4082 \mathrm{eV})$ levels. The basic design is similar to that of Shevchik, ${ }^{13}$ but, rather than a water-cooled glass capillary, a high-temperature material (boron nitride) was used as the discharge capillary. This produced a robust and accident-free source.

The samples were attached to a copper sample holder which was designed to allow for controlled heating using a bifilar wound heater with a thermocouple for control. The samples were cleaned by sputtering with a Varian Model 981-2043 ion bombardment gun, typically at a 


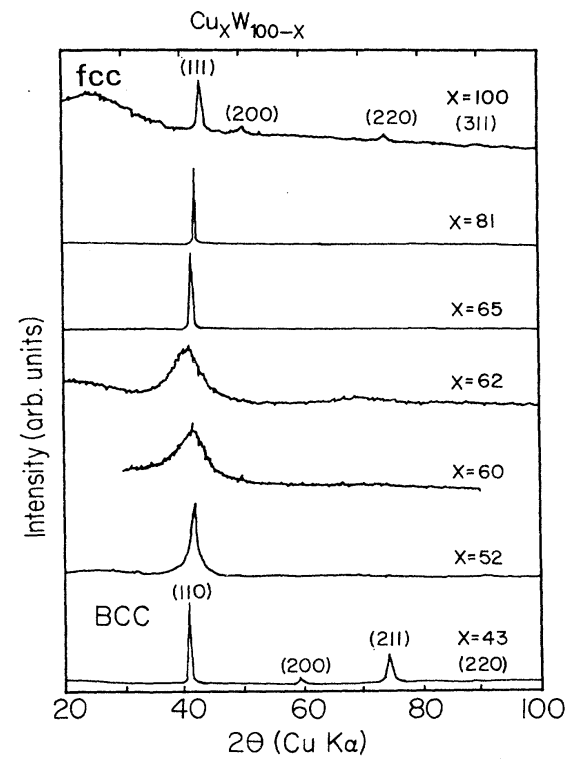

FIG. 1. X-ray diffraction of $\mathrm{Cu}_{x} \mathrm{~W}_{100-x}$ with $x=43-100$.

voltage of $3 \mathrm{kV}$. It is estimated that with a measured current of $31 \mu \mathrm{A} / \mathrm{cm}^{2}$, it took about $1 \mathrm{sec}$ to remove $1 \AA$ of material.

The samples were each typically given a 62 $\mu \mathrm{A} \mathrm{min} / \mathrm{cm}^{2}$ dose $\left(\boldsymbol{P}_{\mathrm{Ar}}=6 \times 10^{-5}\right.$ Torr, 20-mA emission, $2 \mathrm{~min}$ ) to remove any gross contamination. This was done in a continuous fashion by moving each sample into the argon ion beam serially. This precleaning also degassed the ion gun so that degassing would be negligible when final cleaning was performed. Before each PES measurement, the samples were cleaned with an additional $56-\mu \mathrm{A} \mathrm{min} / \mathrm{cm}^{2}$ dose $\left(P_{\mathrm{Ar}}=1 \times 10^{-5}\right.$ Torr, $20-\mathrm{mA}$ emission, $10 \mathrm{~min}$ ).

Contaminations of the samples can be assessed by the

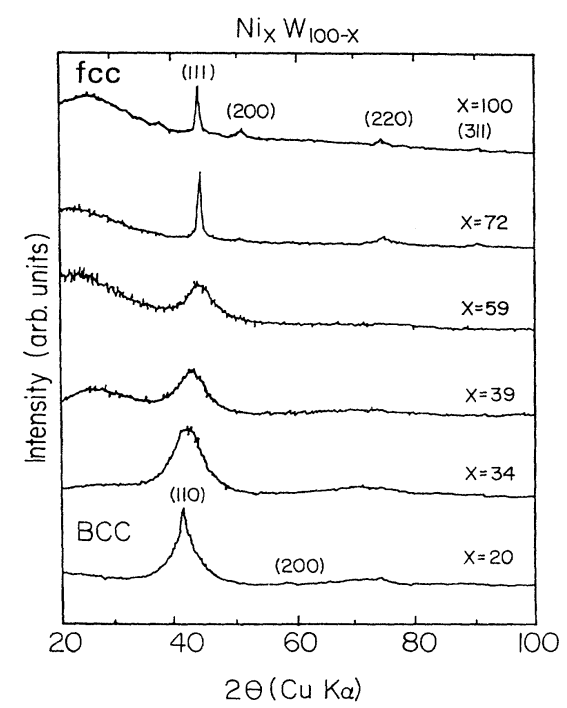

FIG. 2. X-ray diffraction of $\mathrm{Ni}_{x} \mathrm{~W}_{100-x}$ with $x=20-100$.

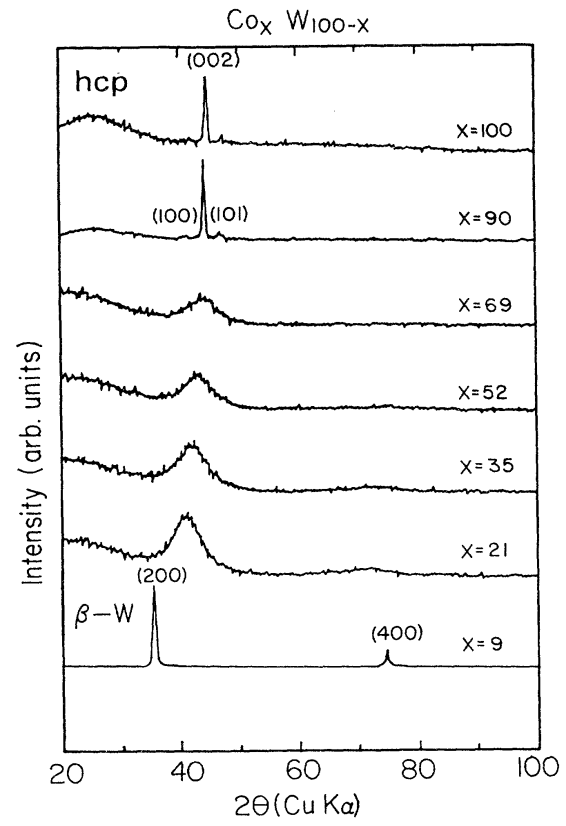

FIG. 3. X-ray diffraction of $\mathrm{Co}_{x} \mathrm{~W}_{100-x}$ with $x=9-100$.

size of the peak at $5-6 \mathrm{eV}$ below the Fermi edge. There are no expected $d$-band spectral features in this range for $\mathrm{W}, \mathrm{Cu}, \mathrm{Ni}, \mathrm{Co}$, or their alloys. However, both oxygen $(2 P$ signal at $-5 \mathrm{eV})$ and carbon monoxide are present as residual gases and as surface contaminants form spectral features in this energy range. A number of cleaning treatments was given to each sample until the -5 to -6-eV peak was either undetectable or at least decreased to a small and constant value.

Auger electron spectroscopy apparatus was not avail-

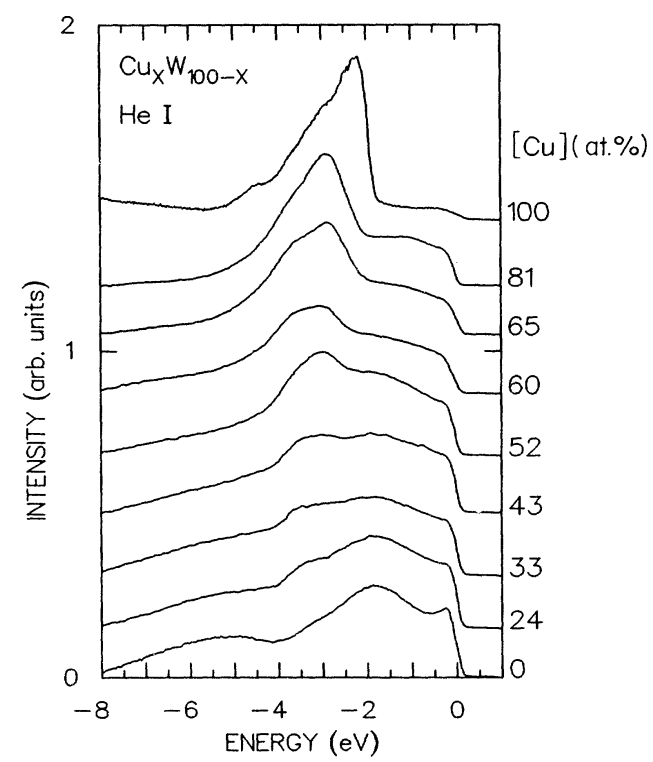

FIG. 4. He I PES data for $\mathrm{Cu}_{x} \mathrm{~W}_{100-x}$. 


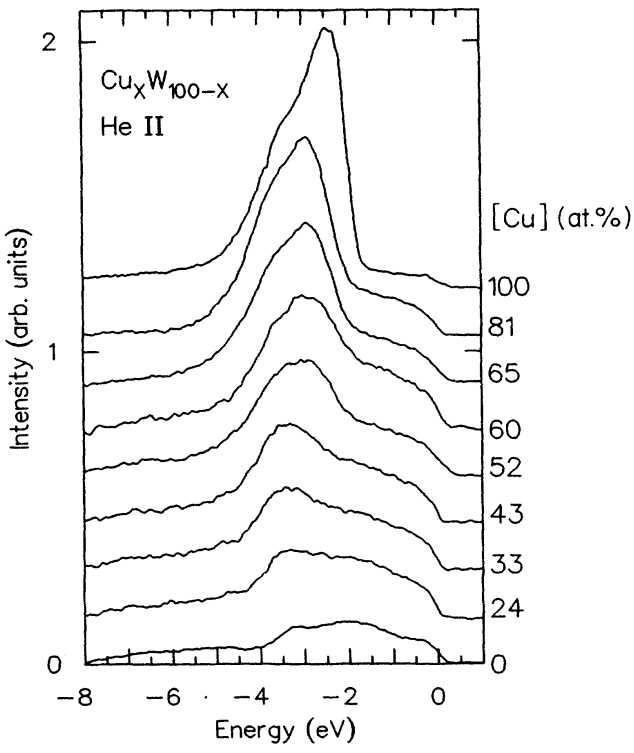

FIG. 5. He II PES data for $\mathrm{Cu}_{x} \mathrm{~W}_{100-x}$.

able for these experiments as a means of checking the surface compositions of the samples. However, for one set of samples $(\mathrm{Cu}-\mathrm{W})$, a study was made of the intensity of the $\mathrm{Cu} 3 d$ peak, which was clearly separable, as a function of composition. Generally these data for $\mathrm{He} \mathrm{I}$ and He II spectra fell on a smooth curve with deviations indicating only a few percent difference on the surface composition from the nominal composition of the alloy. Since we are not comparing absolute intensities with theory, because of matrix-element effects, inter alia, the apparently small compositional uncertainty is not judged

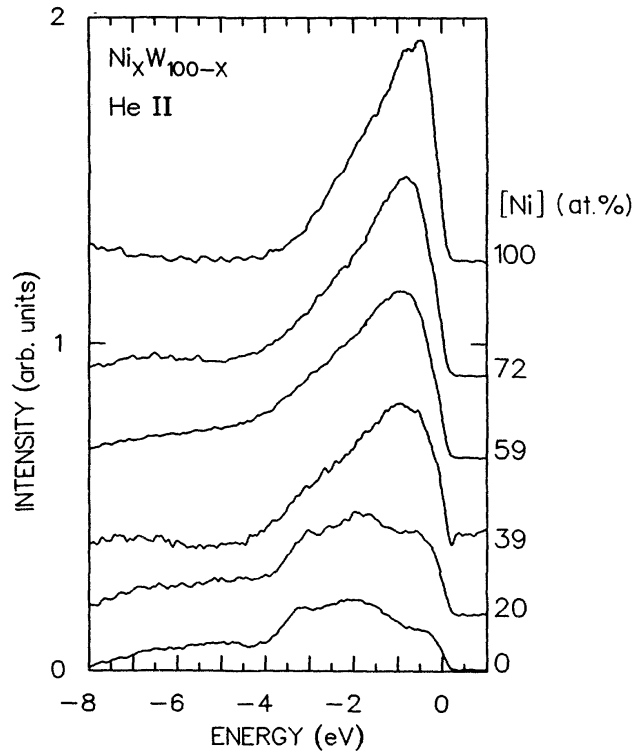

FIG. 7. He II PES data for $\mathrm{Ni}_{x} \mathrm{~W}_{100-x}$.

to be a serious problem.

The photoelectrons were detected with a homemade cylindrical mirror analyzer (CMA) which consists of three concentric cylinders. The properties of our CMA have been discussed in detail by Sar-El. ${ }^{14,15}$ The full width at half maximum of the $\mathrm{He} \mathrm{I}$ excited argon line at $5.46 \mathrm{eV}$ was $0.053 \mathrm{eV}$, indicating a resolution $\Delta E / E$ of about $1 \%$. This is in reasonable agreement with the widths of Fermi edges measured in metallic samples with the system. Further details on the PES apparatus and procedures have been given by Engelhardt. ${ }^{16}$

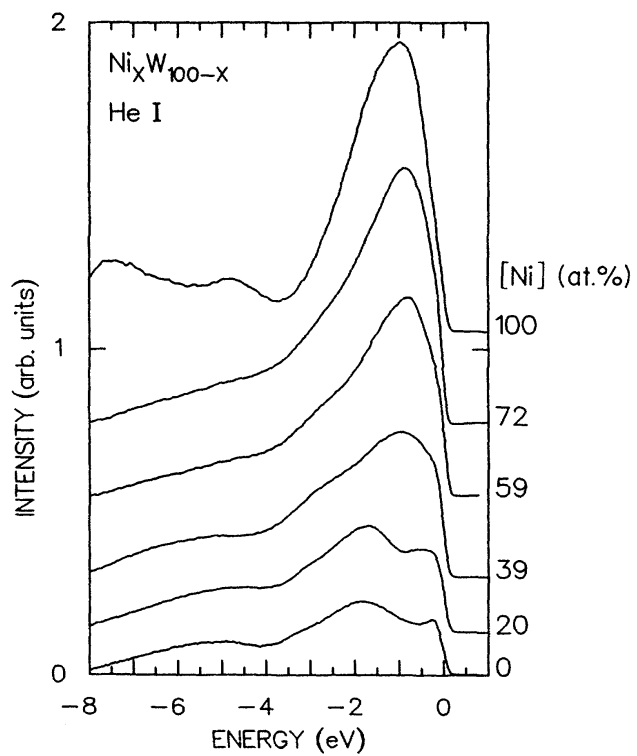

FIG. 6. He I PES data for $\mathrm{Ni}_{x} \mathrm{~W}_{100-x}$.

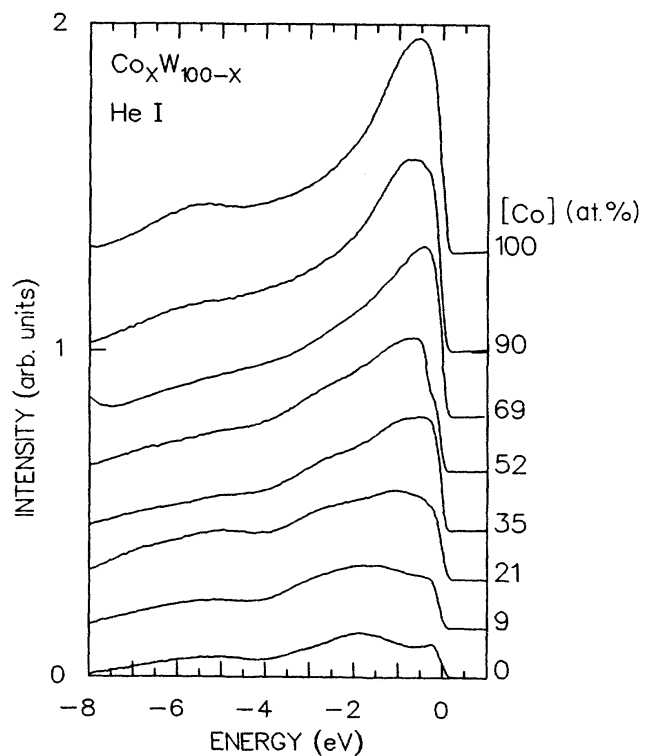

FIG. 8. He I PES data for $\mathrm{Co}_{x} \mathrm{~W}_{100-x}$. 


\section{EXPERIMENTAL RESULTS}

$\mathrm{X}$-ray diffraction scans with $\mathrm{Cu} K \alpha$ radiation on selected samples are shown in Figs. 1-3 for $\mathrm{Cu}_{x} \mathrm{~W}_{100-x}$, $\mathrm{Ni}_{x} \mathrm{~W}_{100-x}$, and $\mathrm{Co}_{x} \mathrm{~W}_{100-x}$, respectively. Because of the large number of samples prepared, scans are shown generally only across the composition region where there is a transition from bcc (W-rich) to fcc (Cu- or Ni-rich) or to hcp (Co-rich). Only rough estimates of the width of the alloy composition for which amorphous alloys form can be obtained from these results. This is because the $\Delta x$ values of the samples typically range from 5 to 10 . Also, any "critical" $x$ value determined in our measurements would not be intrinsic to the alloy system in question because it likely is dependent upon sputtering conditions such as Ar pressure and substrate temperature.

The diffraction data for the $\mathrm{Cu}-\mathrm{W}$ alloys show bcc-like tungsten patterns for $24,33,38,43$, and 52 at. $\% \mathrm{Cu}$; however, the lattice parameters change as do the relative intensities of the peaks as the $\mathrm{Cu}$ concentration increases. Amorphous alloys were found at 60 and 62 at. \% copper. When the copper concentration was increased to 65 and 85 at. $\% \mathrm{Cu}$, the peaks were typical of copper with a shortened lattice parameter. No intermetallic compounds were found for the $\mathrm{Cu} / \mathrm{W}$ series.

Figure 2 shows the $x$-ray diffraction patterns for the NiW series. The sample with 20 at. \% Ni shows vestiges of the tungsten peaks and the next three compositions are approximately amorphous. The sample with 72 at. \% Ni has the features present in fcc nickel with the $d$ spacing lengthened from 2.03 to $2.08 \AA$ for the $43.5^{\circ}$ peak.

The x-ray diffraction patterns for the cobalt series are presented in Fig. 3. An example of the $\beta$-W diffraction pattern is shown at the bottom of a 9 at. \% Co sample which was deposited as an oriented film with only two diffraction lines visible. The four samples from 21 to 69 at. \% Co were all amorphous. At 90 at. \% Co, the structure of hexagonal $\alpha$-Co appears which continued into the pure cobalt film.

The PES He I and He II spectra are presented in Figs. 4-9 for the three alloy series. The He I PES spectrum for tungsten shows a single peak at $E_{B}=-1.85 \mathrm{eV}$ and a feature at the Fermi edge. This Fermi edge feature was suppressed if the surface was contaminated by oxygen $(2 p$ signal at $\sim-5 \mathrm{eV}$ ). The He II spectrum for tungsten shows two features at binding energies of -2.0 and -3.2 eV. The structure at the Fermi edge is no longer resolved. These results are similar to the $\mathrm{x}$-ray photoemission spectroscopy results of White et al. ${ }^{17}$ The three $3 d$-band metals have only single maxima.

The He I spectrum of pure copper has the major $d$ band peak at $E_{B}=-2.2 \mathrm{eV}$ for $\mathrm{He} \mathrm{I}$ photons and at $E_{B}=-2.5 \mathrm{eV}$ for He II photons. In both cases, the $d$ bands are filled from $E_{B}=-2.0$ to $-5.0 \mathrm{eV}$. Nickel and cobalt both have a structure similar to copper with the Fermi level lying in the $d$ bands.

Upon alloying with tungsten, the peak positions move with respect to the Fermi edge. The $\mathrm{CuW}$ alloys display the largest shift with the copper band moving from $E_{B}=-2.2$ to $-2.9 \mathrm{eV}$ after alloying with $20 \%$ copper. The $\mathrm{W}$ peaks at $E_{B}=-2.2$ and $-3.4 \mathrm{eV}$ appear relative-

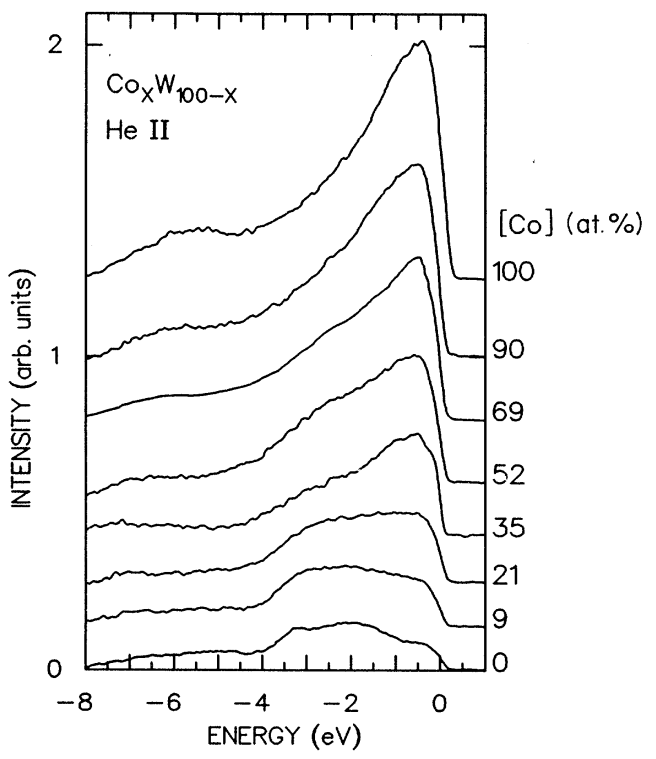

FIG. 9. He II PES data for $\mathrm{Co}_{x} \mathrm{~W}_{100-x}$.

ly unchanged.

The NiW alloys have a different behavior than the $\mathrm{CuW}$ alloys. The $\mathrm{Ni} d$-band peak for the $\mathrm{He}$ I excitation remains stationary. In the pure $\mathrm{Ni}$ spectrum, it appears that there is a slight oxygen contamination on the surface of the sample as evidenced by the small peak at about -5 $\mathrm{eV}$. The $\mathrm{He}$ II photoemission $\mathrm{Ni} 3 d$ peak is centered at about $-0.7 \mathrm{eV}$. On alloying with $\mathrm{Ni}$ the $\mathrm{W} 5 d$ photoelectron peaks moved 0.1 to $0.3 \mathrm{eV}$ higher in energy.

The CoW alloys have a behavior similar to the nickel alloys. Here, the Co $3 d$ band does not move with composition when $\mathrm{He} \mathrm{I}$ is used. With $\mathrm{He}$ II excitation, the W peaks show a small shift towards the Fermi edge, as Co is alloyed with W.

The CuW alloys exhibit a significant energy shift of the $\mathrm{Cu} 3 d$ bands while for $\mathrm{Ni}$ and $\mathrm{Co}$, the movement of the $3 d$ levels was small or negligible. The $\mathrm{W}$ peaks were not affected by alloying with $\mathrm{Cu}$. When $\mathrm{W}$ was alloyed with $\mathrm{Ni}$ or $\mathrm{Co}$, the $\mathrm{W}$ bands shifted slightly. Apparently, the fact that $\mathrm{Cu}$ has a closed $d$ shell while the $\mathrm{Ni}$ and $\mathrm{Co} d$ shells are open significantly affects the properties of the alloy.

\section{ELECTRONIC-STRUCTURE CALCULATIONS AND DISCUSSION}

Electronic-structure calculations were carried out on selected compositions of $\mathrm{Cu}-\mathrm{W}$ and $\mathrm{Ni}-\mathrm{W}$ alloys because they exhibited interesting and distinct features in their photoemission spectra. The Co-W alloys were not included in this study because their data are qualitatively similar to those of Ni-W.

The disorder in the atomic structure is simulated by a random distribution of the constituent atoms in a supercell containing 60 to 64 atoms. The lattice parameters of the crystalline disordered alloys are determined from the $\mathrm{x}$-ray data. The mass densities of the amorphous alloys 


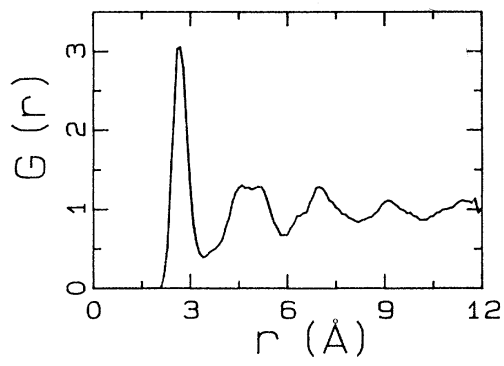

FIG. 10. Total calculated pair-distribution function for the $\mathrm{Cu}_{60} \mathrm{~W}_{40}$ glass.

are unknown and are taken to be $2 \%$ lower than their average values for the constituent atoms. This is a typical change of density for alloys of this class. The amorphous structures are relaxed by Lennard-Jones potentials $\left[V(r)=-A / r^{6}+B / r^{12}+C r+D\right]$. The potential parameters for like pairs were determined from the cohesive energies and lattice constants of the respective crystals. The truncation distances where potentials and their first derivatives vanish were set equal to $7 \AA$. The parameters for the mixed pairs were calculated by averaging the magnitudes and the positions of the minima in the above potentials. The pair-distribution results for the randomly packed and relaxed models were similar to those obtained from molecular dynamics. ${ }^{18}$ There are no experimental data on the pair distribution functions for the systems studied here. As an example, the total calculated pair distribution function for one of the glasses $\left(\mathrm{Cu}_{60} \mathbf{W}_{40}\right)$ is

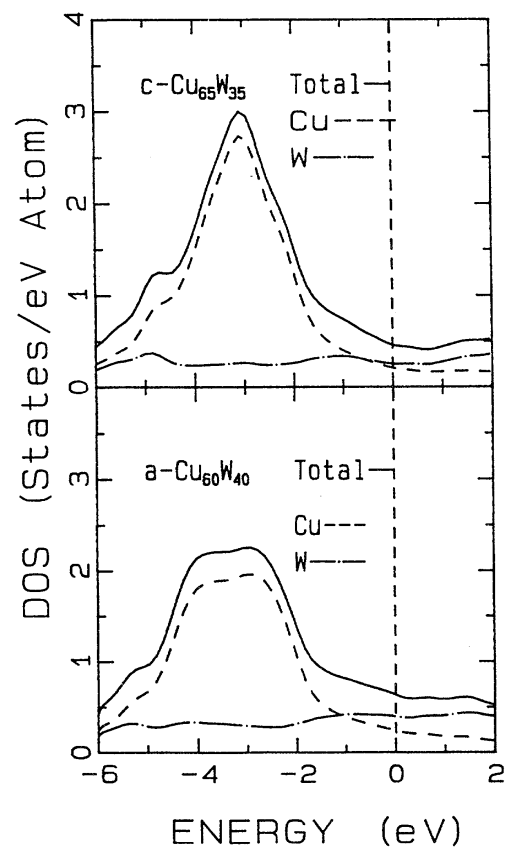

FIG. 11. Total and partial density of states for $c-\mathrm{Cu}_{65} \mathrm{~W}_{35}$ and $a-\mathrm{Cu}_{60} \mathrm{~W}_{40}$.

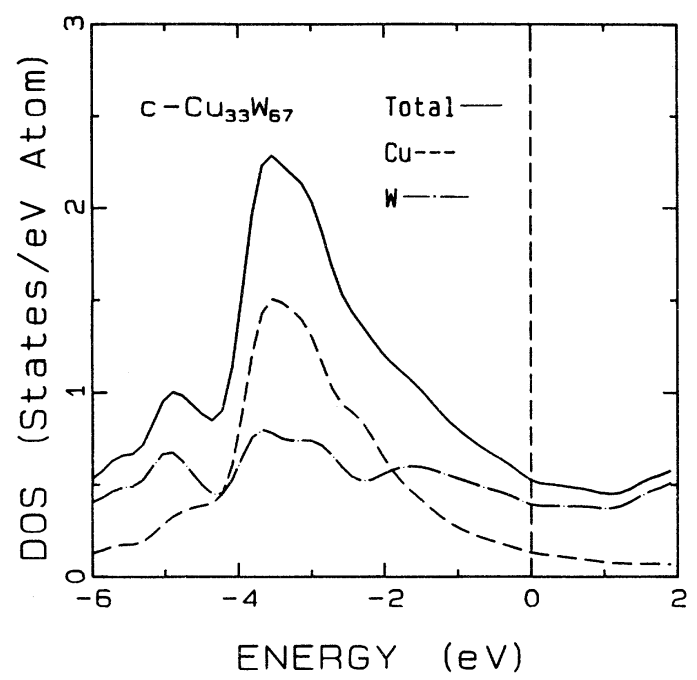

FIG. 12. Total and partial density of states for $c-\mathrm{Cu}_{33} \mathrm{~W}$...

shown in Fig. 10 without any thermal broadening. This result is typical of a metallic glass with a large main peak at the nearest-neighbor distance followed by smaller peaks corresponding to more distant neighbors. The main peak is sharp because $\mathrm{Cu}$ and $\mathrm{W}$ are comparable in size.

The self-consistent electronic-structure calculations are based on the LMTO method $^{19}$ in the scalar-relativistic and local-density approximations. The exchangecorrelation potential is of Hedin-von Barth form ${ }^{20}$ with Janak parameters. ${ }^{21}$ The core states are frozen to be the

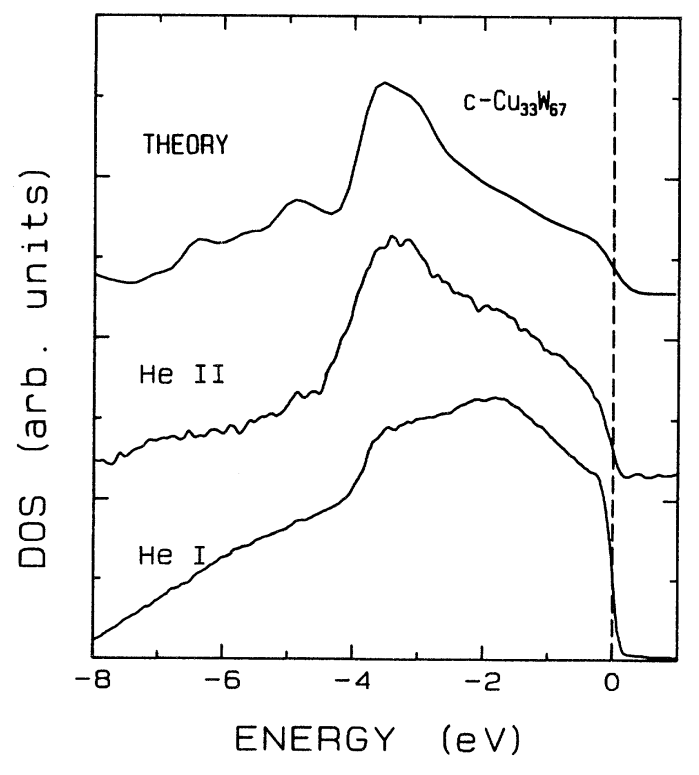

FIG. 13. He I and He II photoemission data and zerotemperature Fermi-function-weighted density of states for $c$ $\mathrm{Cu}_{33} \mathrm{~W}_{67}$. 
same as the atomic states found self-consistently. The $s$, $p$, and $d$ basis functions are used for the valence states of each atom.

$\mathrm{X}$-ray diffraction data given in Fig. 1 show that $\mathrm{Cu}_{65} \mathrm{~W}_{35}$ is disordered face-centered cubic alloy with a lattice parameter of $3.75 \AA$, while $\mathrm{Cu}_{60} \mathrm{~W}_{40}$ is amorphous. The calculated total and partial DOS are shown in Fig. 11. The main contributions to the DOS are from the strongly overlapping $\mathrm{Cu} 3 d$ and $\mathrm{W} 5 d$ bands with the main peak at about $3.5 \mathrm{eV}$ below the Fermi level being due to $\mathrm{Cu} 3 d$ states.

The calculated DOS for every system studied here has a few small peaks at binding energies higher than $4.5 \mathrm{eV}$. In addition to slight variations from system to system, these features are sharper in crystalline alloys than in amorphous alloys. The $\mathrm{W} 6 s$ states are primarily responsible for these peaks. Since the atomic photoionization cross section of the W $6 s$ state is extremely small compared to that of the W $5 d$ state, ${ }^{22}$ this structure appears as a broad feature only in the photoemission data of samples very rich in $\mathrm{W}$, i.e., the $c-\mathrm{Ni}_{20} \mathrm{~W}_{80}$ alloy.

Another aspect that must be kept in mind when comparing calculated DOS and experimental PES data is matrix-element effects in the experimental data. For example, the calculated atomic photoionization cross sections for $\mathrm{Cu} 3 d$ and $\mathrm{W} 5 d$ are in the ratio of about $3: 1$ at $\mathrm{He}$ I energy and $1: 1$ at $\mathrm{He}$ II energy. ${ }^{22}$ Because of the significant localization of $\mathrm{Cu} 3 d$ bands, one can use atomic cross sections to qualitatively explain the differences in the experimental spectra at the two energies, i.e., the $W$ $5 d$ contribution is quite amplified at $\mathrm{He} \mathrm{I}$ compared to that at He II. Also because of the similarity of cross sections, one can better compare the calculated DOS directly with $\mathrm{He}$ II data.

A detailed discussion of the comparison between the calculations shown in Fig. 11 for $c-\mathrm{Cu}_{65} \mathrm{~W}_{35}$ and $a$ $\mathrm{Cu}_{60} \mathrm{~W}_{40}$ has been given previously. ${ }^{9}$ Thus only a sum-

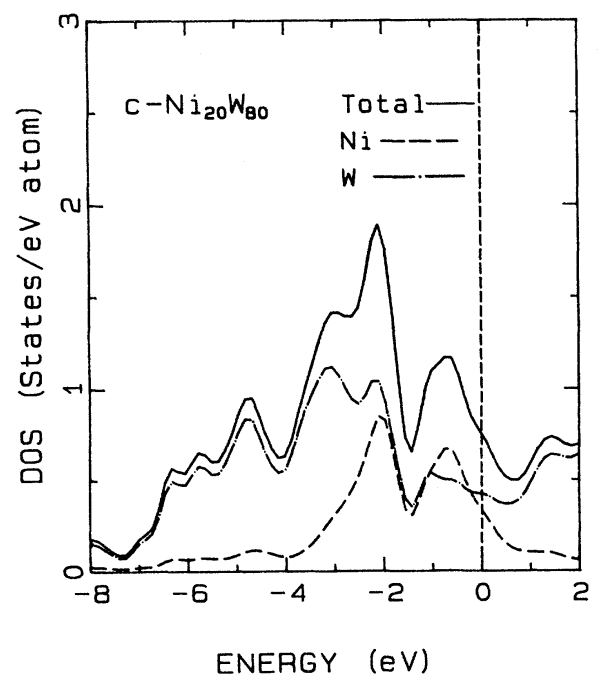

FIG. 14. Total and partial density of states for $c-\mathrm{Ni}_{20} \mathbf{W}_{80}$. mary is given here. It is clear that the calculations do reproduce qualitatively some of the features seen in Figs. 4 and 5 for these compositions. In particular the positions of the $\mathrm{Cu} 3 d$ levels are about right. A major feature of the shape of the $\mathrm{Cu} 3 d$ bands in the calculations is that the crystalline peak is mountain shaped with a single peak, while the amorphous peak has a flattened top or a double-peak feature. This is a reflection of the difference in the long-range structural order for the two systems: the largest $\mathrm{Cu} 3 d$ peak at $-3 \mathrm{eV}$ diminishes in amplitude as one goes from the disordered, fcc alloy to the amorphous system. This is most likely due to a distribution of the near-neighbor distances in the amorphous system. These trends in $\mathrm{Cu} 3 d$ band shape are not as pronounced in the data as predicted by the calculations. The calculated results depend on the mass density, the method used for the electronic-structure calculations, the size of the supercell, and the interatomic potentials used to make the structural model for the amorphous system. The mass density of binary transition-metal glasses is expected to be $2-5 \%$ smaller than the average of the mass density of the constituent metals. The changes in lattice parameter for this range of mass density are too small to have any significant effect on the electronic states. The LMTO method is known to work well for the close-packed metals and this is supported by the present results for the crystalline disordered systems and the amorphous systems studied before. ${ }^{4}$ Increasing the size of the supercell to about one hundred atoms per unit cell and or averaging the results over three or four structural models may help, but the main problem here is the lack of structural data needed to devise interatomic potentials for realistic models.

The third composition chosen for calculations is the $\mathrm{W}$-rich $c-\mathrm{Cu}_{33} \mathrm{~W}_{67}$ alloy. X-ray data show it to be a disor-

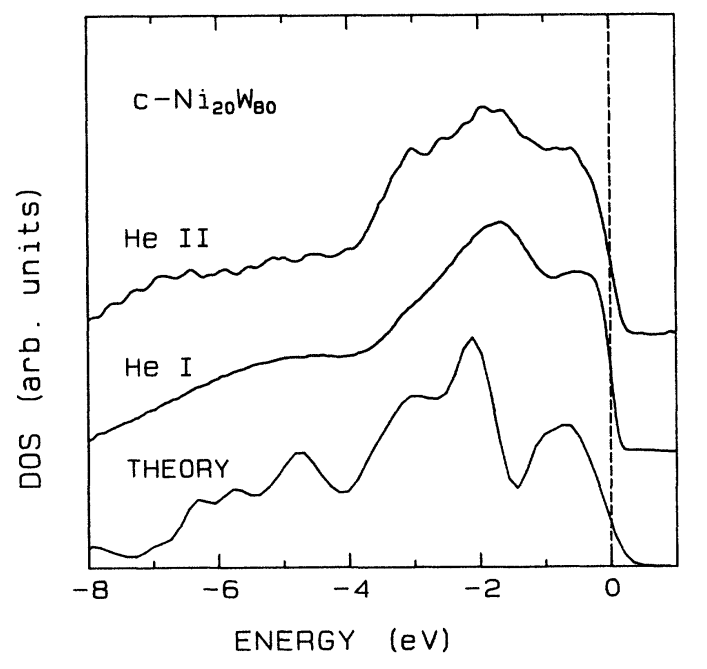

FIG. 15. He I and He II photoemission data and zerotemperature Fermi-function-weighted density of states for $c$ $\mathrm{Ni}_{20} \mathbf{W}_{80}$. 


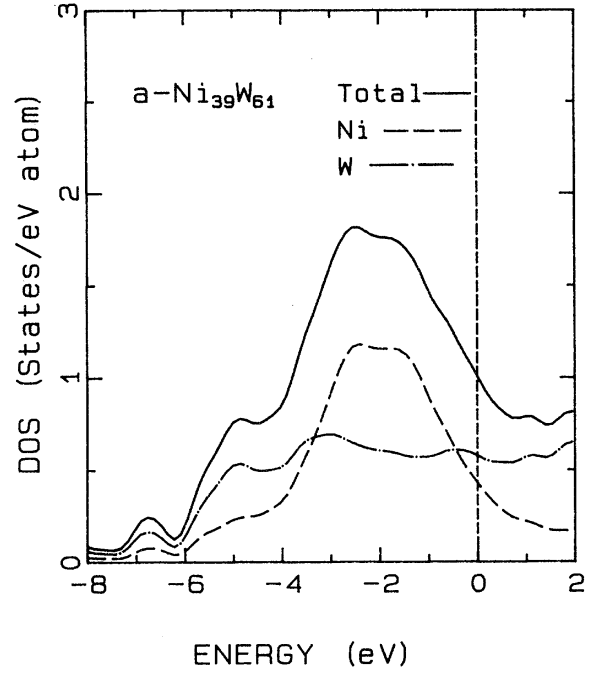

FIG. 16. Total and partial density of states for $a-\mathrm{Ni}_{39} \mathrm{~W}_{61}$.

dered body-centered-cubic alloy with a lattice parameter of $3.07 \AA$. The total and partial DOS are shown in Fig. 12. The DOS is weighted with the zero-temperature Fermi function and then Gaussian broadened with a broadening of $0.2 \mathrm{eV}$. The resulting DOS is compared with the experimental data in Fig. 13. Once again, due to the cross-section effect mentioned above, the calculated DOS compares more favorably with the He II than with He I data, and for the former case the agreement is reasonably good.

From the photoemission spectra given in Figs. 6 and 7, we find the W-rich spectra the most interesting in the Ni-W system. Therefore we have chosen two W-rich

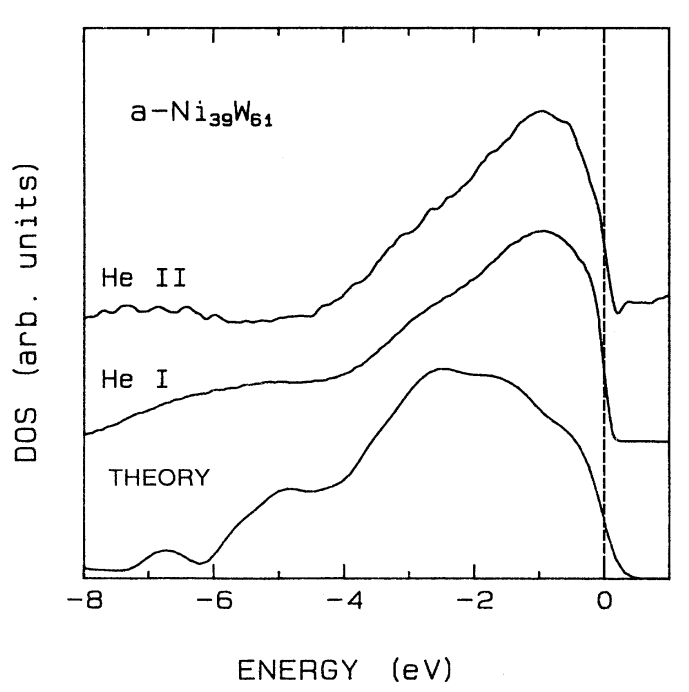

FIG. 17. He I and He II photoemission data and zerotemperature Fermi-function-weighted density of states for $a$ $\mathrm{Ni}_{39} \mathrm{~W}_{61}$. compositions for the electronic-structure calculations. $c$ $\mathrm{Ni}_{20} \mathrm{~W}_{80}$ is a disordered body-centered cubic alloy with a lattice parameter of $3.13 \AA$, while $a-\mathrm{Ni}_{39} \mathrm{~W}_{61}$ is amorphous.

The partial and total DOS for $c-\mathrm{Ni}_{20} \mathrm{~W}_{80}$ are shown in Fig. 14. The DOS is quite similar to that of pure $W$ because $\mathrm{W} 5 d$ and $6 s$ bands dominate over the $\mathrm{Ni} 3 d$ band. ${ }^{23,24}$ The Fermi-function-weighted DOS is compared with the experimental data in Fig. 15. The main peak positions from experiment and theory are in good agreement with each other. The structure at binding energies higher than $4.5 \mathrm{eV}$ has a large contribution from W $6 s$ states and shows up only weakly in the experiment as discussed earlier in this section.

As the $\mathrm{Ni}$ concentration increases, the Ni-W system becomes amorphous and we have studied the electronic structure of $a-\mathrm{Ni}_{39} \mathrm{~W}_{61}$. The total and partial DOS for this system are shown in Fig. 16. The rich structure in DOS of the $c-\mathrm{Ni}_{20} \mathrm{~W}_{80}$ has been replaced by a basically structureless broad band. The $\mathrm{W} 5 d$ band is a uniform background with shallow undulations and a broad Ni $3 d$ band dominates over it. This is an expected change as one goes from a crystalline to an amorphous structure. The Fermi-function-weighted DOS is compared with the experimental data in Fig. 17. In terms of being broad and featureless, the experimental and theoretical spectra agree with each other. They differ in that the calculated $\mathrm{Ni} 3 d$ band has a larger binding energy than that from the experiment. Some of this difference may be due to the structural model used. Detailed experimental information on the structure would be helpful in understanding this difference.

\section{SUMMARY AND CONCLUSIONS}

From the $x$-ray diffraction studies it was found that the amorphous structure range was $52<x<65$ for the $\mathrm{Cu}_{x} \mathrm{~W}_{100-x}$ series, $30<x<50$ for the $\mathrm{Ni}_{x} \mathrm{~W}_{100-x}$ series, and $15<x<80$ for the $\mathrm{Co}_{x} \mathrm{~W}_{100-x}$ series. These rough limits on the range naturally refer only to alloys prepared by cosputtering under the particular conditions employed in this work. The atomic radii for $\mathrm{Co}, \mathrm{Ni}, \mathrm{Cu}$, and $\mathrm{W}$ are $1.25,1.24,1.28$, and $1.41 \AA$, respectively. Thus there is a tendency for a smaller range of amorphousness when the atomic radii of the two elements are closer (e.g., $\mathrm{Cu}$ and W). This trend is consistent with the atomic size model ${ }^{25}$ for predicting the composition range of amorphous alloys.

Because of cross-section effects the photoemission data are sensitive mainly to the $d$ states of the constituents rather than the $s$ and $p$ states. In addition, the He II spectra are generally better for comparison with theoretical density-of-states calculations because the cross sections of the $d$ electrons of the different elements are similar at this photon energy.

The calculated DOS are in very good overall agreement with the photoemission data for crystalline disordered alloys. The trends in the data suggest that there is less difference in the crystalline and amorphous DOS (at 
similar compositions) than is predicted by the calculations. This may be due to the inaccuracies of the pair potentials used to determine the amorphous structures. Further understanding of the differences between theory and experiment will have to be based on accurate diffraction measurements on amorphous systems so that realistic structural models can be developed and put into the electronic-structure calculations. It is hoped that the present work will stimulate such measurements for the present class of intertransition-metal glasses.

\section{ACKNOWLEDGMENTS}

We are grateful for experimental assistance and helpful discussions to J. A. R. Samson, R. D. Kirby, D. Billesbach, K. Aylesworth, Y. G. Ren, and C. Moore. For financial support we are indebted to the National Science Foundation under Grants Nos. DMR-8918889, DMR8605367, and INT-8715441; to the Dale Electronics Corporation, Norfolk, Nebraska; and to the Alcoa Corporation, Pittsburgh, Pennsylvania.
${ }^{*}$ Present address: Synchrotron Radiation Center, University of Wisconsin, Stoughton, WI 53589.

${ }^{1} \mathrm{P}$. Oelhafen, in Glassy Metals II, edited by H. Beck and H. -J. Guntherodt (Springer-Verlag, New York, 1983), Vol. 53, p. 283.

${ }^{2}$ F. W. Saris, L. S. Hung, J. Nastasi, J. W. Mayer, and B. Whitehead, Appl. Phys. Lett. 46, 646 (1985).

${ }^{3}$ J. E. Oh, J. A. Woollam, K. D. Aylesworth, D. J. Sellmyer, and J. J. Pouch, J. Appl. Phys. 60, 4281 (1986).

${ }^{4}$ K. D. Aylesworth, S. S. Jaswal, M. A. Engelhardt, Z. R. Zhao, and D. J. Sellmyer, Phys. Rev. B 37, 2426 (1988).

${ }^{5}$ W. W. Lee, J. Appl. Phys. 42, 4366 (1971).

${ }^{6}$ P. Petroff, T. T. Sheng, A. K. Sinha, G. A. Rozgonyi, and F. B. Alexander, J. Appl. Phys. 44, 2545 (1973).

${ }^{7}$ H. Itoh, T. Moriya, and M. Kashiwagi, Solid State Technol. 30, 83 (1987).

${ }^{8}$ R. Murray, J. Appl. Phys. 58, 3583 (1985).

${ }^{9}$ S. S. Jaswal, M. A. Engelhardt, and D. J. Sellmyer, Bull. Am. Phys. Soc. 34, 555 (1989); M. A. Engelhardt, S. S. Jaswal, and D. J. Sellmyer, Solid State Commun. 75, 663 (1990).

${ }^{10} X$-Ray Powder Data File and Index (American Society for Testing Materials, Philadelphia, 1960).

${ }^{11}$ Crystal Data Determinative Tables Vol. II: Inorganic Compounds (U.S. Department of Commerce, National Bureau of
Standards, Washington, D.C., 1973).

${ }^{12}$ S. Basavaiah and S. R. Pollack, J. Appl. Phys. 39, 5548 (1968).

${ }^{13}$ N. J. Shevchik, J. Electron Spectrosc. Relat. Phenom. 14, 411 (1978).

${ }^{14}$ H. Z. Sar-El, Rev. Sci. Instrum. 38, 1210 (1967).

${ }^{15}$ H. Z. Sar-El, Rev. Sci. Instrum. 42, 1601 (1971).

${ }^{16}$ M. A. Engelhardt, Ph.D. thesis, University of Nebraska, 1988.

${ }^{17}$ R. C. White, C. S. Fadley, M. Sagurton, and Z. Hussain, Phys. Rev. B 34, 5226 (1986).

${ }^{18}$ R. J. Hardy and S. S. Jaswal, Bull. Am. Phys. Soc. 35, 303 (1990).

${ }^{19}$ O. K. Andersen, Phys. Rev. B 12, 3060 (1975); H. L. Skriver, in The LMTO Method, edited by M. Cardona, P. Fulde, and H. J. Queisser, Solid State Sciences Vol. 41 (Springer, New York, 1984).

${ }^{20}$ L. Hedin and V. von Barth, J. Phys. C 5, 1629 (1972).

${ }^{21}$ J. F. Janak, Phys. Rev. B 12, 1257 (1975).

${ }^{22}$ J. J. Yeh and I. Lindau, At. Data. Nucl. Tables 32, 1 (1985).

${ }^{23}$ N. Christensen and B. Feuerbacher, Phys. Rev. B 10, 2349 (1974).

${ }^{24}$ D. M. Bylander and L. Kleinman, Phys. Rev. B 29, 1534 (1984).

${ }^{25}$ S. H. Liou and C. L. Chien, Phys. Rev. B 35, 2443 (1987). 